\title{
Platooning Maneuvers in Vehicular Networks: a Distributed and Consensus-Based Approach
}

\author{
Stefania Santini Member, IEEE, Alessandro Salvi, \\ Antonio Saverio Valente, Antonio Pescapè Senior Member, IEEE, \\ Michele Segata Member, IEEE, Renato Lo Cigno Senior Member, IEEE
}

\begin{abstract}
Cooperative driving is an essential component of future intelligent road systems. It promises greater safety, reducing accidents due to drivers distraction, improved infrastructure utilization, and fuel consumption reduction with platooning applications. Proper platoon management requires Inter-Vehicular Communication (IVC), longitudinal control and lateral control for stability and safety, and proper application protocols protocols and algorithms to manage platoons and perform coordinated maneuvers. This work shows how a longitudinal controller based on distributed consensus can at the same time guarantee stability and performance in regime platoon operation and be at the hearth of maneuvering protocols and algorithms, as it remains stable in face of changes of platoon topology and control gains. The adoption of a single control algorithm for two fundamental tasks greatly simplify the overall design of the system and improves stability and safety as it is not required to switch between different controllers during platoon operation. The theoretical properties are proven in the first part of the paper. The second part of the paper is devoted to its implemented in a state-of-theart mobility and IVC simulator, which is used for an extensive experimental campaign showing the dynamic properties of the system and its performance in a set of typical platoon maneuvers as join, leave and inclusion of a vehicle in the middle of the platoon. All simulations include realistic details of the vehicle dynamics (mass, dimensions, power train dynamics) as well as extremely detailed modeling of the communication network, from 802.11p protocol details, to collisions, packet errors, path loss and fading on the channel, and source-destination based delays.
\end{abstract}

\section{INTRODUCTION}

Cooperative driving can improve road safety, increase the infrastructure efficiency, and reduce fuel consumption [1][9]. Among many cooperative driving applications, platooning targets semi-autonomous driving by having a leading vehicle that drives (it is irrelevant if the driver is a person or a computer) a group of vehicles that automatically follows. The engine, brakes, and steering wheel of followers are controlled with an on-board system whose inputs are local sensors and information received via Inter-Vehicular Communication (IVC) from the leader and from surrounding vehicles, relieving the

A. S. Valente, and A. Pescapè are with University of Napoli Federico II, Italy, Email: \{antoniosaverio.valente, pescape\}@unina.it

S. Santini is with University of Napoli Federico II, Italy, and also with CNR - Italian National Research Council, Institute for Research on Engines (IM), Italy, Email: \{stefania.santini\}@unina.it

A. Salvi is with R\&D department, NetCom Group S.p.A., Naples 80143, Italy, Email: $\{$ a.salvi $\} @$ netcomgroup.eu

M. Segata and R. Lo Cigno are with the University of Trento, Italy, E-Mail: msegata@disi.unitn.it, locigno@disi.unitn.it

The work has been partially funded by the Regione Campania with the PIATTAFORMA MOBILITÀ [CUP B68C12000460007] project. driver from the need for steering, accelerating, and braking. Platooning requires longitudinal (acceleration and braking) and lateral (steering) control, as well as management protocols that supervise the formation of the platoon, maneuvers as well as its disengaging if necessary, as it is clear that the control of the vehicles cannot be returned to a human driver (or an autonomous computer) without proper safety conditions.

In this work we are concerned with longitudinal control, achieved implementing a so called Cooperative Adaptive Cruise Control (CACC) [10]-[14]. Obviously, this is a component of a complete architecture that combines the steering and the longitudinal controllers for ensuring the simultaneous control of longitudinal and lateral motions and all the other components already mentioned. This approach, often proposed in the technical literature, has the advantage to decouple the problems of lateral path, inter-vehicular gaps and speed tracking, in order to cope with the control design for each objective separately (e.g., see [15]-[17] and references therein). Within this decoupled architecture the longitudinal controller we propose is able to opportunely regulate velocity and longitudinal relative distance of each vehicle not only during regime operation, but also during joining and departing maneuvers, while the lateral controller (e.g., [18]) coexists with the CACC without interference.

The duty of the CACC is to decide whether to accelerate or brake to maintain a desired inter-vehicle gap based on local information as well as information obtained from nearby vehicles via IVC. The principle is similar to the one of a standard Adaptive Cruise Control (ACC), a feature that is already installed in modern cars that automatically maintains a desired speed and, thanks to a radar, automatically brakes when approaching a slower vehicle in front. The difference between an ACC and a CACC lies in the information exchange via IVC, as the term "cooperative" clearly indicates. Exploiting information about vehicles out of the radar line-of-sight, a CACC can maintain an inter-vehicle gap as small as a few meters [10], [19], and at such small distances vehicles can save a large amount of fuel thanks to air drag reduction, as experimentally shown in the SARTRE project [2], and improve the infrastructure usage reducing congestion and avoiding to build additional, expensive lane or roads.

Different controller designs exploit different control topologies, i.e., the pattern of information defining what information is used by each vehicle. The CACC developed in the California PATH's project [10], [20] exploits a leader- and predecessorfollowing control topology, meaning that the CACC uses the 
information received from the leading and the front vehicle The CACC designed by Ploeg et.al. [11], instead, uses the data of the front vehicle only. In previous work [12], [21] we designed and evaluated a consensus-based CACC where the control topology is configurable at run-time and not fixed at design-time. The benefit of this approach is that the controller can be re-configured at run-time to match the network and the platoon characteristics, while with a fixed approach a CACC might become unstable (and thus unsafe) if expected data packets are not received due to communication impairments.

The CACC takes care of the platoon once it is formed. Another fundamental issue are the platooning maneuvers, i.e., how to create, merge, and disengage platoons; how vehicles can join or leave, and how to deal with malfunctions. In general, performing maneuvers requires to develop dedicated protocols and controllers for transient phases (e.g, see [22] and references therein for a survey on the topic). In this work, we show how the controller we developed in [12], [21] can be used with any management protocol to perform platooning maneuvers without requiring additional controllers for the transient phases. Therefore, there is no need of switching among different controllers, that may induce loss of stability in the longitudinal closed-loop dynamics [23]. Given this flexibility we show that, by simply changing the parameters at run-time, the controller can naturally maintain longitudinal string stability and performance during maneuvers.

The contributions of the paper are thus the following:

- We formally prove the stability of the controller when the topology changes to accommodate new vehicles or disengage them;

- We give a constructive method to change the consensus parameters at run-time to implement these maneuvers;

- We implement the resulting protocols and controllers in PLEXE [24], and run an extensive experimental campaign to show the performance of join and disengage platooning maneuvers.

\section{Scenario, Motivation, AND Related Work}

Maneuvers are a fundamental component of platooning. Platoons need to be created, maintained, split, joined, and finally taken apart, while vehicles must be able to join or leave a platoon at any time. As for longitudinal control, we need a controller that commands the vehicles' movements and IVC protocols to share the information required to perform the maneuver.

Some examples of a control-theoretic approach include [10], [25], [26]. In these works, the authors define the controllers needed to implement the maneuver and the required high-level communication primitives. In [25] the considered maneuver is a join between two platoons, while in [10] a leave maneuver for a single car.

The work in [26] analyzes different communication patterns to exchange data during a maneuver. More interestingly, the paper defines a set of controllers each one dedicated to a particular functionality as, for example, automated following or maneuvering. This is in contrast with our work, where the same control algorithm can be used both for car-following and for maneuvering.
Some works focus more on protocol definition [27], [28]. In [27] each vehicle is assigned a specific role in the maneuver, and this eases the definition of the protocol. Indeed, this is a good protocol design pattern, which is also used in other works even if its importance is not clearly stressed [10]. The authors of [28], instead, define a protocol to coordinate the information exchange within vehicles in a platoon, showing the effectiveness of the approach in a merging maneuver between two platoons.

Another very interesting and important aspect in platoon maneuvering is fault detection or emergency behaviors. During cruising, or while performing a maneuver, the system must detect and deal with problems that include mechanical faults [29], [30], network failures [31], and interference from non-automated vehicles [32]-[34]. In [30] the authors develop a fault detection system focusing on mechanical and electronic faults of devices such as speed sensors, brake actuators, etc. Upon this, the work in [29] builds a set of control actions that can be taken as countermeasures thanks to a dedicated network protocol used for coordination. In [34], the authors propose a set of maneuvers following an agent-based approach that takes into account non-automated vehicles as well. However, no guarantee on the performance of the system is given, which, besides the way we model the problem, is the main difference with our work.

A work resembling our philosophy is [31]. The authors show that, in absence of the control signal provided by means of wireless communication, their CACC can safely use an estimate computed by the radar. In this case there is a single control law that changes its characteristics depending on the input, rather than changing the control law itself. Their approach, however, only focuses on car-following and does not consider maneuvering.

Finally, the works in [32]-[35] deal with interference from human-driven vehicles and emergencies. As an example, [32] considers a join maneuver where a vehicle joins the platoon in the middle. During this maneuver vehicles leave space for the new one to join, but at that point a human-driven vehicle might enter the gap creating a potentially dangerous situation. The paper describes protocols and methods to detect and counteract such situations, but to actually perform the maneuver the authors need to tweak the CACC they consider.

This background work clearly witnesses the importance of maneuvering for a platooning application but, to the best of our knowledge, there is no work on a single automated controller that can support longitudinal stability during a maneuver by design. In this work we theoretically prove the stability of our control approach for maneuvering and show its performance by means of realistic simulations through the PLEXE framework [24], which is a state of the art simulation framework dedicated to cooperative driving based on Veins [36], [37]. In particular, we consider three maneuvers:

1) simple platoon split, where we re-configure the controller at run-time to show basic stability properties;

2) join at back, where a vehicle joins a platoon as the last vehicle; and

3) join at middle, where a vehicle joins in the middle of a platoon requiring the vehicles to create a gap. 
We test each scenario in different dynamic conditions, i.e. at constant speed, under sinusoidal disturbance, and under intermittent sinusoidal disturbance. Finally, we test the behavior of the third maneuver in a realistic network setup, where other vehicles generate interference by using the same wireless channel.

\section{CONSENSUS-BASED MANEUVERING WITH A SWITCHING NETWORKS APPROACH}

\section{A. Mathematical Preliminaries and Nomenclature}

A graph is used to model the inter-vehicle communication structure, where every vehicle is a node. A platoon of $N$ vehicles can be modeled as a directed graph (digraph) $\mathcal{G}=(\mathcal{V}, \mathcal{E}, \mathcal{A})$ of order $N$, where $\mathcal{V}=\{1, \ldots, N\}$ is the set of nodes and $\mathcal{E} \subseteq \mathcal{V} \times \mathcal{V}$ is the set of edges. The graph topology is described by an adjacency matrix with non-negative elements $\mathcal{A}=\left[a_{i j}\right]_{N \times N}$ : we consider $a_{i j}=1$ the presence of a communication link from node $j$ to node $i$, otherwise $a_{i j}=0$; moreover, we assume self-edges $(i, i)$ are not allowed, i.e., $a_{i i}=0$. The edge $(i, j) \in \mathcal{E}$ is used to indicate that vehicle $i$ can obtain information from vehicle $j$ and uses it for platooning, but not necessarily vice versa.

The $N$ vehicles follow a leader vehicle labelled with index zero, i.e., node 0 . An augmented directed graph $\overline{\mathcal{G}}$ is used to model the platoon topology based on the communication pattern desired by the consensus algorithm. Node 0 is globally reachable in $\overline{\mathcal{G}}$ if there is a path in $\overline{\mathcal{G}}$ from every node $i$ in $\mathcal{G}$ to node 0 [38].

To model changing topologies due to maneuvers we introduce a switching signal $\sigma(t):[0, \infty) \rightarrow \phi_{\Gamma}=\{1,2, \ldots, G\}$ that determines the network topology. We denote by $\Gamma=$ $\left\{\overline{\mathcal{G}}_{1}, \overline{\mathcal{G}}_{2}, \ldots, \overline{\mathcal{G}}_{G}\right\}$ a finite collection of graphs with a common node set $\overline{\mathcal{V}}$ describing all the possible topologies that can be obtained by varying the use of information by each vehicle [39]. $G$ is the total number of all possible digraphs, and cooresponding adjacency matrices, describing all the possible stages within platoon manouvers, while $\sigma(t)$ determines the index of the active graph at time instant $t$. Note that $\sigma(t)$ is piecewise constant and continuous from the right. Moreover, we assume that two consecutive switching instants are separated by some finite dwell-time. This guarantees that the switching frequency remains bounded so that Zeno behavior cannot occur, [40].

Before proceeding to design our consensus controller, we recall here some useful definitions and lemmas.

Definition 1. A complex square matrix is said to be negative stable [positive stable] if its spectrum lies in the open left [right] half of the complex plane [41].

Definition 2. Let $Z_{N}=\left\{C=\left(c_{i j}\right)_{N \times N} \in M_{N}(\mathbb{R}): c_{i j} \leq\right.$ $0 \quad$ if $i \neq j, \quad i, j=1,2, \ldots, N\}$, where $M_{N}(\mathbb{R})$ denotes the set of all $N \times N$ matrices with entries from $\mathbb{R}$. A matrix $C=\left(c_{i j}\right)_{N \times N}$ is said to be an M-matrix if $C \in Z_{N}$ and $C$ is positive stable [42].

Lemma 1. [42] If $C \in Z_{N}$, the following statements are equivalent :

1) $C$ is positive stable, that is, $C$ is an M-matrix.
2) All principal minors of $C$ are positive.

3) The diagonal entries of $C$ are positive and $C \tilde{D}$ is strictly row diagonally dominant for some positive diagonal matrix $\tilde{D}$.

4) $C$ is non-singular and $C^{-1}$ is a non-negative matrix.

Definition 3. [43] A matrix $C=\left(c_{i j}\right)_{N \times N} \in M_{N}(\mathbb{R})$ is a weakly chained diagonally dominant (WCCD) matrix if $C$ is diagonally dominant, that is

$$
\left|c_{i j}\right| \geq \sum_{i=1, j \neq i}^{N}\left|c_{i j}\right|, \quad i \in \mathcal{V}_{N}, \mathcal{V}_{N}=\{1,2, \ldots, N\}
$$

and

$$
J(C)=\left\{i \in \mathcal{V}_{N}:\left|c_{i i}\right|>\sum_{i=1, j \neq i}^{N}\left|c_{i j}\right|\right\} \neq \emptyset
$$

for each $i \in \mathcal{V}_{N}, i \notin J(C)$, there is a sequence of non-zero elements of $C$ of the form $c_{i, i_{1}}, c_{i_{1}, i_{2}}, \ldots, c_{i_{r}, j}$ with $j \in J(C)$.

Lemma 2. [44] Let $C \in Z_{N}$ and $C$ be a WCCD matrix, then $C$ is an M-matrix. Furthermore, assume $C \in Z_{N}$ and

$$
c_{i i} \geq \sum_{i=1, i \neq j}^{N}\left|c_{i j}\right|, \quad i=1,2, \ldots, N
$$

then $C$ is an M-matrix if and only if $C$ is a WCCD matrix.

\section{B. Control Design}

The goal of the platoon longitudinal control is to regulate the speed of each vehicle based on the leader speed, and its relative distance from the vehicle in front. Hence, a platoon is composed by a string of $N$ vehicles plus the additional leading vehicle acting as a reference for the string. In our analysis each vehicle is equipped with on-board sensors to measure its absolute position, speed and acceleration, while IVC based on IEEE 802.11 p radio technology enables sharing information among all vehicles with broadcast beacons.

The generic $i$-th vehicle dynamic is described by the following inertial agent $(i=1, \ldots, N)$ :

$$
\begin{aligned}
& \dot{r}_{i}(t)=v_{i}(t) \\
& \dot{v}_{i}(t)=\frac{1}{M_{i}} u_{i}(t),
\end{aligned}
$$

where $r_{i}[\mathrm{~m}]$ and $v_{i}[\mathrm{~m} / \mathrm{s}]$ are the $i$-th vehicle absolute position (with respect to a given reference framework) and speed; $M_{i}$ [kg] is the $i$-th vehicle mass; the propelling force $u_{i}$ is the control input to be appropriately chosen to achieve the control goal. Similarly, the leader vehicle dynamics are

$$
\begin{aligned}
& \dot{r}_{0}(t)=v_{0} ; \\
& \dot{v}_{0}=0 .
\end{aligned}
$$

being $r_{0}$ and $v_{0}$ the leader state variables. Given Eqs. (4) and (5), the problem of maintaining a desired inter-vehicle spacing policy and a common speed can be rewritten as the following high-order consensus problem:

$$
\begin{aligned}
r_{i}(t) & \rightarrow \frac{1}{\Delta_{i}}\left\{\sum_{j=0}^{N} a_{i j} \cdot\left(r_{j}(t)+d_{i j}\right)\right\} \\
v_{i}(t) & \rightarrow v_{0} .
\end{aligned}
$$

where $d_{i j}$ is the desired distance between vehicles $i$ and $j$; $a_{i j}$ (for $i=1, \ldots, N$ and $j=0, \ldots, N$ ) models the platoon 
topology defined by the inclusion/exclusion of a communication link between vehicles $i$ and $j ; \Delta_{i}=\sum_{j=0}^{N} a_{i j}$ is the degree of vehicle/agent $i$, i.e., the number of vehicles establishing a communication link with vehicle $i$. Note that according to [45] the desired spacing $d_{i j}$ can be expressed as $d_{i j}=h_{i j} v_{0}+d_{i j}^{s t}$, where $h_{i j}$ is the constant time headway (i.e., the time necessary to vehicle $i$-th to travel the distance to its predecessor), and $d_{i j}^{s t}$ is the distance between the vehicles $i-$ th and $j-$ th at standstill. In a real world setting, the precision of the distance measurement can be improved by complementing the GPSbased distance with local radar measures. In this work, however, we consider GPS positions to be correct but to become outdated due to delays in the transmission process. Furthermore we remark that $a_{i j}$ are the non-negative elements of the adjacency matrix associated to the platoon topology directed graph $\overline{\mathcal{G}}$; $a_{0 j}=0(\forall j=0, \ldots, N)$, since the leader does not consider data from any other vehicle.

The platoon problem in Eq. (6) is solved here by the following decentralized control action embedding the spacing policy information as well as all the time-varying communication delays:

$$
\begin{array}{r}
u_{i}=\quad-b\left[v_{i}(t)-v_{0}\right]+\frac{1}{\Delta_{i}} \sum_{j=0}^{N} k_{i j} a_{i j}\left[h_{i j} v_{0}+d_{i j}^{s t}\right] \\
-\frac{1}{\Delta_{i}} \sum_{j=0}^{N} k_{i j} a_{i j}\left[r_{i}(t)-r_{j}\left(t-\tau_{i j}(t)\right)-\tau_{i j}(t) v_{0}\right]
\end{array}
$$

where $k_{i j}$ and $b$ are control gains to be appropriately tuned to achieve the consensus positions and speeds; $\tau_{i j}(t)$ and $\tau_{i 0}(t)$ are the unavoidable time-varying communication delays when information is transmitted to vehicle $i$ from vehicle $j$ and from the leader respectively (in general $\tau_{i j}(t) \neq \tau_{j i}(t)$ ). Each control link is hence characterized individually: each node is characterized by a different number of edges, each one being affected by a different time-varying delay $\tau_{i j}(t)$. All delays are bounded in $0 \leq \tau_{i j}(t) \leq \tau$. This assumption is not restrictive for the considered technological scenario. Indeed, the communication is performed through local broadcast packets, which, given characteristics of the 802.11p MAC, implies that information is delivered within few hundreds microseconds (see [46] and references therein) or it is lost. An information beacon can be lost due to collision or it may be discarded because a new beacon arrives before it is transmitted, thus, naturally $\tau \leq 100 \mathrm{~ms}$, which is the beacon generation time foreseen for platooning applications.

\section{Closed-loop Dynamics}

In this section we analytically prove the closed-loop stability of the platoon. The proof of stability is based on the recast of the closed-loop dynamics as a set of functional differential equations for which it is possible to find a quadratic LyapunovRazumikhin function [47].

To this goal, we define position and speed errors with respect to the reference signals $r_{0}(t), v_{0}(i=1, \ldots, N)$ as:

$$
\begin{aligned}
& \bar{r}_{i}=\left(r_{i}(t)-r_{0}(t)-h_{i 0} v_{i}-d_{i 0}^{s t}\right) ; \\
& \bar{v}_{i}=\left(v_{i}(t)-v_{0}\right) .
\end{aligned}
$$

Re-writing the coupling control action $u_{i}$ in terms of the state errors $\bar{r}_{i}$ and $\bar{v}_{i}$ and expressing headway constants $h_{i j}$ and standstill distances $d_{i j}^{s t}$ with respect to the leading vehicle, namely $h_{i j}=h_{i 0}-h_{j 0}$ and $d_{i j}^{s t}=d_{i 0}^{s t}-d_{j 0}^{s t}$, after some algebraic manipulation the closed-loop dynamics can be rewritten as $(i=1, \ldots, N)$ :

$$
\left\{\begin{array}{l}
\dot{\bar{r}}_{i}=\bar{v}_{i}, \\
M_{i} \dot{\bar{v}}_{i}=-\frac{1}{\Delta_{i}}\left(k_{i 0} a_{i 0}+\sum_{j=1}^{N} k_{i j} a_{i j}\right) \bar{r}_{i}-b \bar{v}_{i}(t)+ \\
\quad+\frac{1}{\Delta_{i}} \sum_{j=1}^{N} k_{i j} a_{i j}\left[\bar{r}_{j}\left(t-\tau_{i j}(t)\right)\right] .
\end{array}\right.
$$

To describe the platoon dynamics in presence of the timevarying delays in a more compact form we define the position and speed error vectors as $\bar{r}=\left[\bar{r}_{1}, \ldots, \bar{r}_{i} \ldots, \bar{r}_{N}\right]^{\top}, \bar{v}=$ $\left[\bar{v}_{1}, \ldots, \bar{v}_{i} \ldots, \bar{v}_{N}\right]^{\top}$, and the error state vector as $\bar{x}(t)=$ $\left[\bar{r}^{\top}(t) \bar{v}^{\top}(t)\right]^{\top}$. Moreover delays $\tau_{i j}$ in Eq. (9) can be recast as $\left.\tau_{p}(t) \in\left\{\tau_{i j}(t): i, j=1,2, \ldots, N, i \neq j\right)\right\}$ for $p=$ $1,2, \ldots, m$ with $m \leq N(N-1)\left(0 \leq \tau_{p}(t) \leq \tau\right)$. Note that $m$ is equal to its maximum, $N(N-1)$, if the platoon topology is represented by a directed complete graph and all time delays are different.

According to the above definitions, the closed loop platoon dynamics can be represented as the following set of functional differential equations:

$$
\dot{\bar{x}}(t)=A_{0} \bar{x}(t)+\sum_{p=1}^{m} A_{p} \bar{x}\left(t-\tau_{p}(t)\right),
$$

where $m$ is the total number of different time delays and

$$
A_{0}=\left[\begin{array}{cc}
0_{N \times N} & I_{N \times N} \\
-M \widetilde{K} & -M \widetilde{B}
\end{array}\right] \quad \text { and } \quad A_{p}=\left[\begin{array}{cc}
0_{N \times N} & 0_{N \times N} \\
M \widetilde{K}_{p} & 0_{N \times N}
\end{array}\right]
$$

being

$$
\begin{gathered}
M=\operatorname{diag}\left\{\frac{1}{M_{1}}, \ldots, \frac{1}{M_{N}}\right\} \in \mathbb{R}^{N \times N} \\
\widetilde{B}=\operatorname{diag}\{b, \ldots, b\} \in \mathbb{R}^{N \times N} ; \\
\widetilde{K}=\operatorname{diag}\left\{\tilde{k}_{11}, \ldots, \tilde{k}_{N N}\right\} \in \mathbb{R}^{N \times N}, \text { with } \tilde{k}_{i i}=\frac{1}{\Delta_{i}} \sum_{j=0}^{N} k_{i j} a_{i j} ;
\end{gathered}
$$

and $\tilde{K}_{p}=\left[\bar{k}_{p i j}\right] \in \mathbb{R}^{N \times N}(p=1, \ldots, m)$ the matrix defined according to the formalism adopted in [18] as:

$$
\bar{k}_{p i j}=\left\{\begin{array}{cc}
\frac{a_{i j} k_{i j}}{\Delta_{i}}, & j \neq i, \tau_{p}(\cdot)=\tau_{i j}(\cdot), \\
0, & j \neq i, \tau_{p}(\cdot) \neq \tau_{i j}(\cdot) . \\
0, & j=i .
\end{array}\right.
$$

Taking into account the changing topologies that arise during maneuvers the closed-loop dynamic system Eq. (10) can be expressed, according to [48], as the following switched timedelayed system:

$$
\dot{\bar{x}}(t)=A_{0, \sigma} \bar{x}(t)+\sum_{p=1}^{m} A_{p, \sigma} \bar{x}\left(t-\tau_{p}(t)\right),
$$

where $\sigma$ is the switching signal, defined as in Sec. III-A, and

$$
\begin{aligned}
& A_{0, \sigma}=\left[\begin{array}{cc}
0_{N \times N} & I_{N \times N} \\
-M \widetilde{K}_{\sigma} & -M \widetilde{B}
\end{array}\right], \\
& A_{p, \sigma}=\left[\begin{array}{cc}
0_{N \times N} & 0_{N \times N} \\
M \widetilde{K}_{p, \sigma} & 0_{N \times N}
\end{array}\right],
\end{aligned}
$$


whose solutions are defined in the sense of Caratheodory [49] System (16) can be written in compact form as [39]:

$$
\dot{\bar{x}}(t)=F_{\sigma} \bar{x}(t)-\sum_{p=1}^{m} C_{p, \sigma} \int_{-\tau_{p}(t)}^{0} \bar{x}(t+s) d s,
$$

where

$$
C_{p, \sigma}=A_{p, \sigma} A_{0, \sigma}=\left[\begin{array}{cc}
0_{N \times N} & 0_{N \times N} \\
0_{N \times N} & M \widetilde{K}_{p, \sigma}
\end{array}\right],
$$

and

$$
F_{\sigma}=A_{0, \sigma}+\sum_{p=1}^{m} A_{p, \sigma}=\left[\begin{array}{cc}
0_{N \times N} & I_{N \times N} \\
-M \widehat{K}_{\sigma} & -M \widetilde{B}
\end{array}\right],
$$

with

$$
\widehat{K}_{\sigma}=-\sum_{p=1}^{m} \widetilde{K}_{p, \sigma}+\widetilde{K}_{\sigma}
$$

Let $\mathcal{I}=\left\{i \mid k_{i 0} a_{i 0}>0, i \in \mathcal{V}_{N}\right\}$ denote the index set of the vertices whose neighbors include vertex 0 , then the following Lemma holds.

Lemma 3. Assume $k_{j i}>0$ such that

$$
\begin{aligned}
& \sum_{j=1}^{N} k_{i j} a_{i j} \geq \sum_{j=1}^{N} k_{j i} a_{j i}, \quad i \notin \mathcal{I}, i \in \mathcal{V}_{N} ; \\
& 2 k_{i 0} a_{i 0}+\sum_{j=1}^{N} k_{i j} a_{i j}>\sum_{j=1}^{N} k_{j i} a_{j i}, \quad i \in \mathcal{I} ;
\end{aligned}
$$

and that vertex 0 is globally reachable in $\overline{\mathcal{G}}_{\sigma}$.

Then $H_{\sigma}+H_{\sigma}^{\top}=\left(\bar{h}_{i j}\right)_{N \times N}$ with $H_{\sigma}=M \widehat{K}_{\sigma}$ is positive definite.

Proof: $H_{\sigma}+H_{\sigma}^{\top} \in Z_{N}$ is symmetric, hence, $H_{\sigma}+H_{\sigma}^{\top}$ is positive definite if and only if it is an M-matrix. From assumptions (22), it follows that $H_{\sigma}+H_{\sigma}^{\top}$ is diagonally dominant, that is

$$
\bar{h}_{i i} \geq \sum_{i=1, j \neq i}^{N}\left|\bar{h}_{i j}\right|, \quad i \in \mathcal{V}_{N}
$$

and

$$
\begin{aligned}
& \left|\bar{h}_{i i}\right|=2\left(\frac{1}{M_{i} d_{i}} k_{i 0} a_{i 0}+\frac{1}{M_{i} d_{i}} \sum_{j=1}^{N} k_{i j} a_{i j}\right)> \\
& \frac{1}{M_{i} d_{i}} \sum_{j=1}^{N} k_{i j} a_{i j}+\frac{1}{M_{i} d_{i}} \sum_{j=1}^{N} k_{j i} a_{j i}=\sum_{i=1, j \neq i}^{N}\left|\bar{h}_{i j}\right|, i \in \mathcal{I}
\end{aligned}
$$

According to Lemma 2, $H_{\sigma}+H_{\sigma}^{\top}$ is positive definite if and only if $H_{\sigma}+H_{\sigma}^{\top}$ is a WCCD matrix. Since the vertex 0 is globally reachable in $\overline{\mathcal{G}}_{\sigma}$, then $\mathcal{I} \neq \emptyset$ and for each vertex $i \in \mathcal{V}_{N}, i \notin \mathcal{I}$, a sequence of non-zero elements of the form $\bar{h}_{i, i_{1}}, \bar{h}_{i_{1}, i_{2}}, \ldots, \bar{h}_{i_{r}, j}$ with $j \in \mathcal{I}$ exists; hence $H_{\sigma}+H_{\sigma}^{\top}$ is a WCCD matrix, that implies $H_{\sigma}+H_{\sigma}^{\top}$ to be positive definite.

Now we prove the platoon stability during maneuvers by exploiting Lemma 3 and Lyapunov-Razumikhin functional techniques [47].
Theorem 1. Consider the closed-loop system (18). Assume that hypotheses of Lemma 3 are fulfilled, select gains $k_{i j}$ accordingly, and choose $b$ in the control low such that

$$
b>b_{1}^{\star}=\left\{\frac{\widehat{\mu}}{2 \widehat{\lambda}}+1\right\} M_{i},
$$

with $\widehat{\mu}=\max _{\sigma}\left\{\lambda_{\max }\left(H_{\sigma} H_{\sigma}^{\top}\right)\right\}$ and $\widehat{\lambda}=\min _{\sigma}\left\{\lambda_{\min }\left(H_{\sigma}+\right.\right.$ $\left.\left.H_{\sigma}^{\top}\right)\right\}$.

If there exists a positive definite matrix $P$ and a constant $\tau=\tau_{1}^{\star}$, solutions of the following problem $\forall \sigma=1, \ldots, G$ :

$\tau_{1}^{\star}=\max _{\tau, P} \tau$

subject to :

$$
\left[\begin{array}{cc}
P F_{\sigma}+F_{\sigma}^{\top} P+m \tau q P & \mathcal{S}_{\sigma} \\
\mathcal{S}_{\sigma}^{\top} & \mathcal{T}
\end{array}\right]<0,
$$

with

$$
\mathcal{T}=\operatorname{diag}\left\{-\frac{1}{\tau} P, \ldots,-\frac{1}{\tau} P\right\}, \mathcal{S}_{\sigma}=\left[\begin{array}{llll}
P C_{1, \sigma} & \ldots & P C_{m, \sigma}
\end{array}\right]
$$

then, for all $0 \leq \tau_{p}(t) \leq \tau<\tau_{1}^{\star}(p=1, \ldots, m)$, the origin of the closed loop system is globally asymptotically stable

$$
\lim _{t \rightarrow \infty} \bar{x}(t)=0 .
$$

Proof: Consider the following Lyapunov-Razumikhin candidate function

$$
V(\bar{x})=\bar{x}^{\top} P \bar{x}
$$

with positive definite matrix $(i=1, \ldots, N)$

$$
P=\left[\begin{array}{cc}
b M I_{N \times N} & I_{N \times N} \\
I_{N \times N} & I_{N \times N}
\end{array}\right], \quad \frac{b}{M_{i}}>1 .
$$

For the case of switching topologies, we obtain

$$
\begin{aligned}
& \dot{V}(\bar{x}) \leq \bar{x}^{\top}\left(P F_{\sigma}+F_{\sigma}^{\top} P\right) \bar{x} \\
+ & \sum_{p=1}^{m}\left[\tau_{p}(t) \bar{x}^{\top} P C_{p, \sigma} P^{-1} C_{p, \sigma}^{\top} P \bar{x}\right. \\
+ & \left.\int_{-\tau_{p}(t)}^{0} \bar{x}^{\top}(t+s) P \bar{x}(t+s) d s\right] .
\end{aligned}
$$

By choosing the continuous, non decreasing function $\psi_{4}(s)=$ $q s$ (for some constant $q>1$ ), after some algebraic manipulations, when

$$
V(\bar{x}(t+\theta))<\psi_{4}(V(\bar{x}))=q V(\bar{x}(t)),-\tau \leq \theta \leq 0,
$$

we obtain that inequality (31) becomes

$$
\dot{V}(\bar{x}) \leq-\bar{x}^{\top} Q_{\sigma} \bar{x}+\tau \sum_{p=1}^{m} \bar{x}^{\top}\left(P C_{p, \sigma} P^{-1} C_{p, \sigma}^{\top} P+q P\right) \bar{x}
$$

being

$Q_{\sigma}=-\left(P F_{\sigma}+F_{\sigma}^{\top} P\right)=\left[\begin{array}{cc}H_{\sigma}+H_{\sigma}^{\top} & H_{\sigma}^{\top} \\ H_{\sigma} & 2(M b-1) I_{N \times N} \\ & \end{array}\right]$

According to Lemma $3, H_{\sigma}+H_{\sigma}^{\top}$ is positive definite. Hence, from Schur complement lemma [38], matrix $Q_{\sigma}$ is positive definite if $b$ fulfills conditions (25). 
Exploiting equation (27), inequality (33) can be rewritten as

$\dot{V}(\bar{x}) \leq \bar{x}^{\top}\left[P F_{\sigma}+F_{\sigma}^{\top} P+m \tau q P-\mathcal{S}_{\sigma} \mathcal{T}^{-1} \mathcal{S}_{\sigma}^{\top}\right] \bar{x}$,

Hence, by the Schur complement lemma, supposing to restrict matrix $P$ to the form presented in (30), it is possible to choose the maximum allowable delay $\tau=\tau_{1}^{\star}$ by solving the problem (26). This implies to look for the largest $\tau$, say $\tau_{1}^{\star}$ (by, e.g., bisection), in the interval $\tau \in\left[0, \tau_{\max }\right]$ such that the solution of the problem (26) exists for the positive definite matrix $P$, chosen as presented in (30). If the problem (26) is feasible then the inequality (35) is negative definite, so we can write $\dot{V}(\bar{x}) \leq-\eta \bar{x}^{\top} \bar{x}$ for some constant $\eta>0$. Hence asymptotic stability follows from Lyapunov-Razumikhin Theorem as done in [50].

Note that if the matrix $P$ is not restricted to (30), the delay $\tau_{1}^{\star}$ calculated as solution of (26) would be, in general, larger.

We also remark that the technical Lemma 3 provides practical conditions for the computation of lower bounds that delimit the asymptotic consensus region given the set of the switching topologies. It is clear that, during the implementation phases, an LMI-based procedure solves (e.g., by exploiting Yalmip) inequalities in Lemma 3 and ensures that the delay-dependent stability conditions in Theorem 1 are fulfilled. As usual for tuning procedures, the values of the control gains can be chosen by designers within the consensus region so that additional requirements with respect to the stability conditions can be achieved. Here, according to this approach, we adopted a trialand-error technique to tune the controller gains to achieve good transient performance.

\section{Simulation Scenario And Maneuvers}

The analysis carried out in Sec. III demonstrates stability and convergence given the consensus is always reached during the maneuvers themselves. To verify stable behavior and the dynamic performance we have implemented the proposed approach in the platooning simulator PLEXE [24], which features realistic network and vehicle dynamics. Indeed, PLEXE exploits, in a integrated simulation environment, the network simulator OMNeT++/Veins, the road traffic simulator SUMO and additional vehicle models. OMNeT++/Veins is used to emulate V2V communication based on the 802.11p standard. Cooperative control algorithms can be extensively analyzed in an environment, that mimics realistic wireless network features, transmission delays, and beaconing strategy. It follows that the delays do not have to be artificially modeled through ideal, mathematical functions, but they naturally arise from the specific conditions of each communication link, as happens during on-the-road tests. Further details on the simulation platform can be found in [12]. The control algorithm (Eq. (7)) and the relative information exchange are an extension of PLEXE and will be made available to the community with the next release of the simulator. We have not devised a full protocol for maneuvers, as it is out of the scope of this paper, but we have implemented the actual dissemination of the control topology and parameters encapsulating the control matrix to be used in the periodic beacons sent by the leader, so that we also implicitly explore the robustness of the approach in case some information on the maneuvers is lost or delayed. As mentioned, we assume the existence of a high-level architecture including lateral control and maneuvers protocols and algorithms. Since our focus is on the longitudinal performance, during the simulations the lateral controller has been emulated with the default SUMO lane changing algorithm. In the simulations we use the predecessor- and leader-following topology for the following reasons. First, it is a commonly-assumed flow topology in platooning systems (see [2], [10], [13], [14]). Second, as the presence of a leader is inevitable to coordinate the maneuver, exploiting the data included in its beacon improves the robustness, although the performance of our control system is guaranteed even without such communication link.

The simulations consider three maneuvers. In the first scenario a vehicle performs a join-at-tail and a leave-at-tail maneuver in a 4-car platoon. The control topology that defines the join part is depicted in Fig. 1. The meaning of a red arrow from car $j$ to car $i$ is that "car $i$ uses information from car $j$ " in the consensus algorithm, and thus it is directly related to the adjacency matrices in Tab. I. The combined join and leave maneuver is obtained using the adjacency matrices in Tab. I in the order $1 \rightarrow 2 \rightarrow 3 \rightarrow 2 \rightarrow 1$; when using adjacency matrix 3 the platoon is in a stable operating condition with 5 cars.

The second maneuver is a join-at-middle as in [32]. The control topologies used are represented in Fig. 2. The initial platoon is composed by 4 cars and a fifth car needs to enter the platoon in third position. The first operation is thus to open a space for the joining vehicle, which is performed by telling vehicles in third and fourth position to become the vehicles in fourth and fifth position, respectively and to increase their distance to accomodate the new vehicle. At the same time, we need to change the adjacency matrix. The next step is to disable the link $1 \rightarrow 3$, communicate position 2 to the other vehicles, and enable the links $1 \rightarrow 2,2 \rightarrow 3$ and $0 \rightarrow 2$, ending up with a 5-car platoon using a leader-predecessor topology. We perform the maneuver by switching between the matrices in Tab. II using the following order: $1 \rightarrow 2 \rightarrow 3 \rightarrow 4 \rightarrow 5 \rightarrow 6$.

The last maneuver is a leave-at-middle. The third car in a platoon of five vehicles wants to leave. In this case the third vehicles changes lane to leave and then vehicles four and five close the gap by becoming vehicles three and four respectively. This maneuver is trivially implemented by switching between the matrices in Tab. II in the reversed order of the join in the middle one.

We test each maneuver in different disturbance scenarios. The first scenario considers no disturbance at all, i.e., the leader travels with a constant speed while the maneuver is being performed. In the second scenario the disturbance is intermittent. Immediately after switching adjacency matrix, the leader begins to change speed in a sinusoidal fashion for an amount of time equal to half switch time (20s). In the remaining half switch time, the leader drives with constant speed to let the followers converge before switching to the next adjacency matrix. In the third scenario the leader is constantly changing its speed following a sinusoidal trend. Furthermore, in some scenarios, we reduce the switch time between the adjacency matrices as an additional test on robustness. 
1)

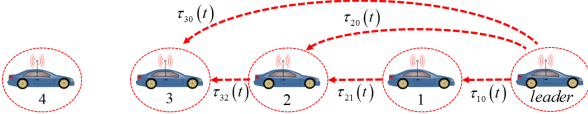

2)

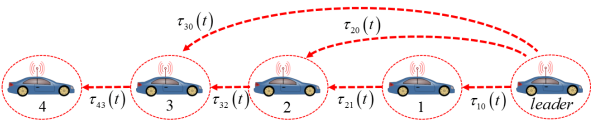

3)

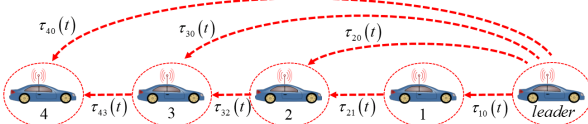

Figure 1. Evolution of the controller information use in the "join at the tail" maneuver.

Table I

ADJACENCY MATRICES FOR TOPOLOGIES RELATED TO THE JOIN AND LEAVE AT THE TAILS MANEUVERS IN FIG. 1.

$\left[\begin{array}{lllll}0 & 0 & 0 & 0 & 0 \\ 1 & 0 & 0 & 0 & 0 \\ 1 & 1 & 0 & 0 & 0 \\ 1 & 0 & 1 & 0 & 0 \\ 0 & 0 & 0 & 0 & 0\end{array}\right]$

Adj. matrix 1

$\left[\begin{array}{lllll}0 & 0 & 0 & 0 & 0 \\ 1 & 0 & 0 & 0 & 0 \\ 1 & 1 & 0 & 0 & 0 \\ 1 & 0 & 1 & 0 & 0 \\ 0 & 0 & 0 & 1 & 0\end{array}\right]$

Adj. matrix 2

$$
\left[\begin{array}{lllll}
0 & 0 & 0 & 0 & 0 \\
1 & 0 & 0 & 0 & 0 \\
1 & 1 & 0 & 0 & 0 \\
1 & 0 & 1 & 0 & 0 \\
1 & 0 & 0 & 1 & 0
\end{array}\right]
$$

Adj. matrix 3

In all the scenarios, the leading vehicle is driven by a standard ACC, while all the followers are controlled by the control algorithm proposed in this work. It is assumed that non-platooning cars do not interfere with maneuvers. Some considerations on this interesting and important topic can be found in [32]

\section{EXPERIMENTAL ANALYSIS}

We investigate the maneuvers described Sec. IV in a realistic network environment, where the platoon performing the maneuver drives on two dedicated lanes (two lanes are needed even for a single platoon for the join-in-middle maneuver). The remaining three lanes (on both directions) are occupied by human-driven vehicles. In the base scenario these cars do not use IVC, while in one final scenario to test robustness in vase of all disturbances together, they send $10 \mathrm{~Hz}$ beacons that disturb the communication channel forcing additional further packet losses. The simulation is first warmed-up for $500 \mathrm{~s}$ to fill the freeway with human-driven vehicles. Next, we inject the platooning vehicles and let the platoon perform the maneuver. Tab. III summarizes the human-driven vehicles parameters, while Fig. 3 shows a screenshot of the simulation setup.

Tab. IV summarizes network simulation parameters. To model the channel we consider a free-space path loss model with Nakagami fading and thermal noise, thus channel errors

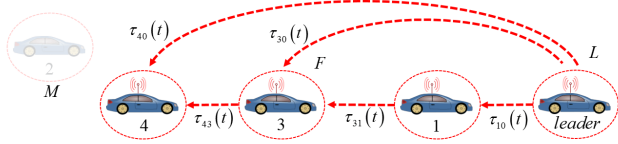

2)
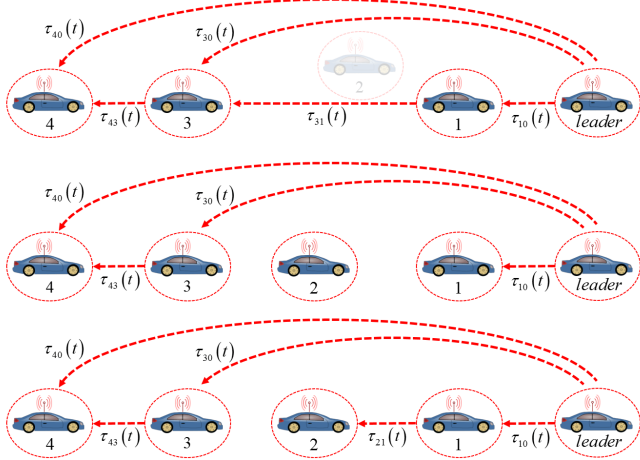

5)
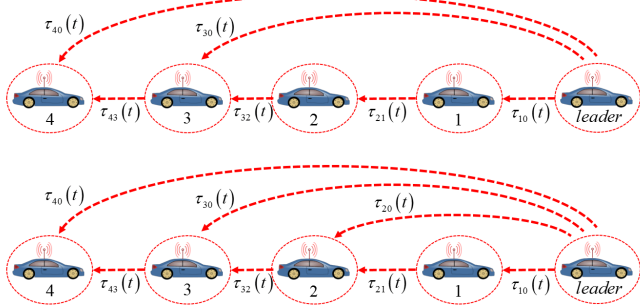

Figure 2. Evolution of the controller information use in the "join in the middle" maneuver.

Table II

ADJACENCY MATRICES FOR TOPOLOGIES RELATED TO THE JOIN AND LEAVE IN THE MIDDLE MANEUVER.

$$
\left[\begin{array}{lllll}
0 & 0 & 0 & 0 & 0 \\
1 & 0 & 0 & 0 & 0 \\
1 & 1 & 0 & 0 & 0 \\
1 & 0 & 1 & 0 & 0 \\
0 & 0 & 0 & 0 & 0
\end{array}\right]
$$

Adj. matrix 1

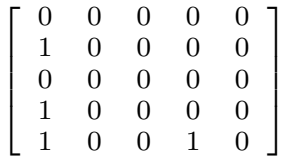

Adj. matrix 3

$$
\left[\begin{array}{lllll}
0 & 0 & 0 & 0 & 0 \\
1 & 0 & 0 & 0 & 0 \\
0 & 1 & 0 & 0 & 0 \\
1 & 0 & 1 & 0 & 0 \\
1 & 0 & 0 & 1 & 0
\end{array}\right]
$$

Adj. matrix 5

$\left[\begin{array}{lllll}0 & 0 & 0 & 0 & 0 \\ 1 & 0 & 0 & 0 & 0 \\ 0 & 0 & 0 & 0 & 0 \\ 1 & 1 & 0 & 0 & 0 \\ 1 & 0 & 0 & 1 & 0\end{array}\right]$

Adj. matrix 2

$\left[\begin{array}{lllll}0 & 0 & 0 & 0 & 0 \\ 1 & 0 & 0 & 0 & 0 \\ 0 & 1 & 0 & 0 & 0 \\ 1 & 0 & 0 & 0 & 0 \\ 1 & 0 & 0 & 1 & 0\end{array}\right]$

Adj. matrix 4

$$
\left[\begin{array}{lllll}
0 & 0 & 0 & 0 & 0 \\
1 & 0 & 0 & 0 & 0 \\
1 & 1 & 0 & 0 & 0 \\
1 & 0 & 1 & 0 & 0 \\
1 & 0 & 0 & 1 & 0
\end{array}\right]
$$

Adj. matrix 6 and collisions are correctly modeled with very good fidelity to real networks. We use a standard IEEE $802.11 \mathrm{p} / 1609.4$ implementation sending frames at $6 \mathrm{Mbit} / \mathrm{s}$. The payload size of each frame is $200 \mathrm{~B}$ and each vehicle sends 10 frames per second. For all maneuvers, we experiment with different holding times $T_{h}$ between matrix switches ranging from $40 \mathrm{~s}$ to 10 s: not even with such unrealistic high switching rate there 


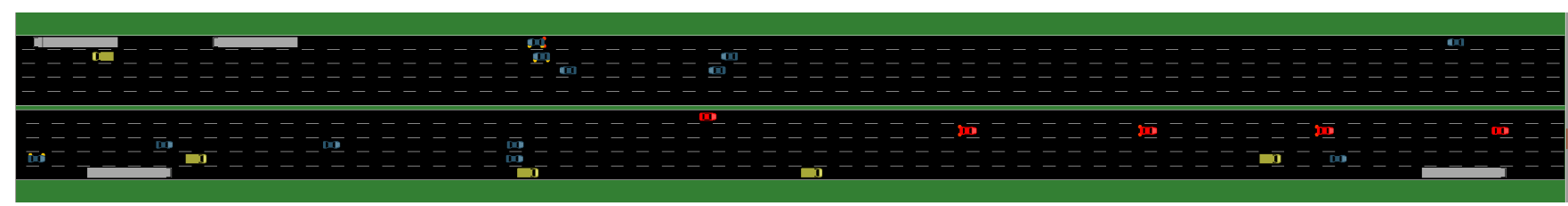

Figure 3. Screenshot of the realistic scenario showing the platoon (red cars) performing the join at the tails maneuver.

Table III

ROAD AND NON-PLATOONING TRAFFIC SIMULATION PARAMETERS; VEHICLES DRIVE ACCORDING TO THE IDEAL DRIVER MODEL AVAILABLE IN SUMO.

\begin{tabular}{lr}
\hline Parameter & Value \\
\hline Freeway length & $10 \mathrm{~km}$ \\
Lanes & 5 (two-way) \\
Cars percentage (length $4 \mathrm{~m}$ ) & $50 \%$ \\
Trucks percentage (length $20 \mathrm{~m})$ & $20 \%$ \\
Vans percentage (length $5 \mathrm{~m})$ & $30 \%$ \\
Inter-vehicle time & $\sim \exp (0.7276 / \mathrm{s})(\mathbb{E}[X]=1.374 \mathrm{~s}$ \\
Cars' speed & $\sim U(100 \mathrm{~km} / \mathrm{h}, 160 \mathrm{~km} / \mathrm{h})$ \\
Trucks' - Vans' speed & $80 \mathrm{~km} / \mathrm{h}-100 \mathrm{~km} / \mathrm{h}$ \\
\hline
\end{tabular}

Table IV

NETWORK SIMULATION PARAMETERS.

\begin{tabular}{lr}
\hline Parameter & Value \\
& Realistic channel \\
Path loss model & Free space $(\alpha=2.0)$ \\
Fading model & Nakagami-m $(m=3)$ \\
PHY/MAC model & IEEE $802.11 \mathrm{p} / 1609.4$ single channel $(\mathrm{CCH})$ \\
Frequency & $5.89 \mathrm{GHz}$ \\
Bitrate & $6 \mathrm{Mbit} / \mathrm{s}(\mathrm{QPSK} \mathrm{R}=1 / 2)$ \\
Access category & AC_VI \\
MSDU size & $200 \mathrm{~B}(\mathrm{byte})$ \\
Transmit power & $20 \mathrm{dBm}$ \\
Beacon frequency & $10 \mathrm{~Hz}$ \\
\hline
\end{tabular}

are signs of instability. The holding time $T_{h}$ is reported in the results' figures captions.

Tab. V lists all control parameters. To show the robustness of our approach with respect to non-homogeneous vehicles, we randomly pick a vehicle mass between $1000 \mathrm{~kg}$ and $2000 \mathrm{~kg}$. In addition, vehicles are subject to an actuation lag modeled by the following first-order filter

$$
\dot{a}_{i}(t)=-\frac{1}{\tau} a_{i}(t)+\frac{1}{\tau} u_{i}(t) .
$$

The time constant $\tau$ is random between $0.2 \mathrm{~s}$ and $0.5 \mathrm{~s}$.

\section{A. Join and Leave at the Tail}

We first consider the join at the tail and leave at the tail maneuvers for the scenario with no leader disturbance (standard consensus). The results (Figs. $4 \mathrm{a}, 4 \mathrm{~d}$ and $4 \mathrm{~g}$ ) show the dynamics of the maneuver in terms of inter-vehicle distances, speeds, and acceleration versus time. At the beginning of the plot we can see the four cars composing the initial platoon stabilizing, while vehicle $V_{4}$ remains at a larger distance at the back of the platoon, as mandated by the human driving model. The maneuver starts around the simulation time of $45 \mathrm{~s}$ when vehicle $V_{4}$ gets notified by the leader to "connect" to vehicle $V_{3} . V_{4}$ thus speeds up and joins the platoon matching cruising speed and following $V_{3}$ at the required gap. The second matrix switch that
Table V

CONTROL PARAMETERS.

\begin{tabular}{lr}
\hline Parameter & Value \\
\hline Mass $M_{i}$ & $\sim U(1000 \mathrm{~kg}, 2000 \mathrm{~kg})$ \\
Actuation lag $\tau$ & $\sim U(0.2 \mathrm{~s}, 0.5 \mathrm{~s})$ \\
Standstill distance $d_{i j}^{s t}$ & $15 \mathrm{~m}$ \\
Headway time $h_{i j}$ & $0.8 \mathrm{~s}$ \\
Gain $b$ & 1800 \\
Gains $k_{0, j}$ & $0,0,0,0,0$ \\
Gains $k_{1, j}$ & $460,0,0,0,0$ \\
Gains $k_{2, j}$ & $80,860,460,460,460$ \\
Gains $k_{3, j}$ & $80,860,860,460,460$ \\
Gains $k_{4, j}$ & $80,860,860,860,460$ \\
Acceleration limits & $-9 \mathrm{~m} / \mathrm{s}^{2}, 1.5 \mathrm{~m} / \mathrm{s}^{2}$ \\
\hline
\end{tabular}

enables the link between the leader and the joining vehicle is not noticeable, and $V_{4}$ stably follows $V_{3} . V_{4}$ accelerations look "sharp" because of the time scale, indeed, they remain within an interval very comfortable for passengers $\left(-3 \mathrm{~m} / \mathrm{s}^{2}\right.$ and $\left.1.5 \mathrm{~m} / \mathrm{s}^{2}\right)$. Finally, when $V_{4}$ decides to leave around simulation time of $160 \mathrm{~s}$, the leader communicate to remove the control links and switch back to the original matrix; $V_{4}$ returns to a standard ACC (or human driving), safely increasing its gap to $V_{3}$ and concluding the maneuver.

Figs. $4 \mathrm{~b}, 4 \mathrm{c}, 4 \mathrm{e}, 4 \mathrm{f}, 4 \mathrm{~h}$ and $4 \mathrm{i}$ show the results for the same maneuver for both scenarios with leader disturbance. This time the plots show the sinusoidal disturbance, but this does not qualitatively affect the results. The maneuver is successfully performed, showing the robustness of topology switching even in case of disturbance.

\section{B. Join at Middle}

Fig. 5 shows the results for the join-at-middle maneuver. The maneuver starts with a platoon composed of four cars $\left(V_{0}\right.$, $V_{1}, V_{3}$, and $V_{4}$ ) traveling on a dedicated lane and the joining vehicle $\left(V_{2}\right)$ traveling on an adjacent lane. At around $50 \mathrm{~s}$, on the leader command, vehicles $V_{3}$ and $V_{4}$ change their position from third to fourth and from fourth to fifth, respectively. This makes them decelerate to correct their position error as if there was a vehicle between $V_{1}$ and $V_{3}$, so that $V_{3}$ measures a double distance from the vehicle in front. Roughly $10 \mathrm{~s}$ later, their position converge to the desired value, so $V_{4}$ follows again $V_{3}$ at roughly $40 \mathrm{~m}$, while $V_{3}$, having left the gap for the joiner, is now $80 \mathrm{~m}$ from $V_{1}$. At $120 \mathrm{~s}$ the leader communicates $V_{2}$ that it is now part of the platoon, and that it should be in third position. This causes $V_{2}$ to speed up and reach the join position. Finally, at $160 \mathrm{~s}, V_{2}$ changes lane and joins the platoon; its distance from the vehicle in front "drops" suddenly from "infinite" to the right distance as it changes lane ${ }^{1} . V_{3}$ and $V_{2}$ distance

\footnotetext{
${ }^{1}$ The sudden change in distance is due to the discrete lane change model
} of SUMO, which changes lane in a single simulation timestep. 


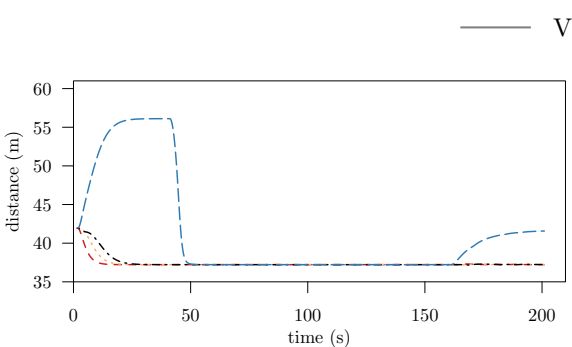

(a)

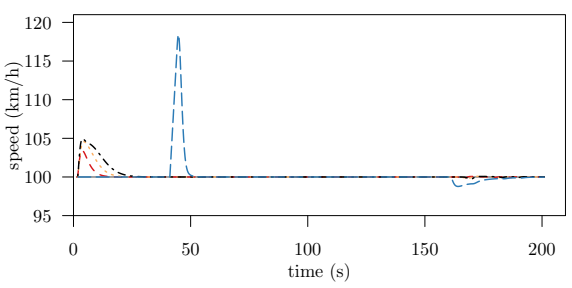

(d)

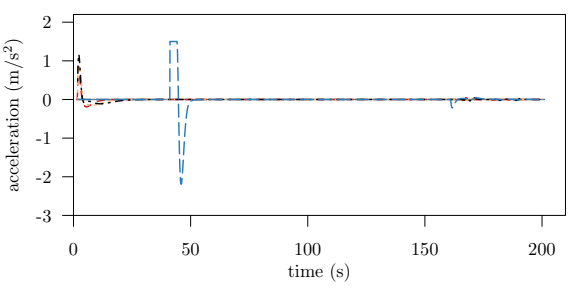

(g)

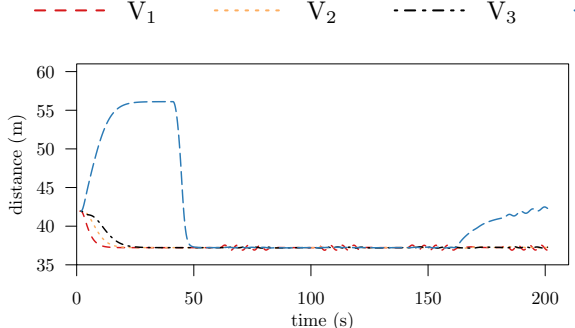

(b)

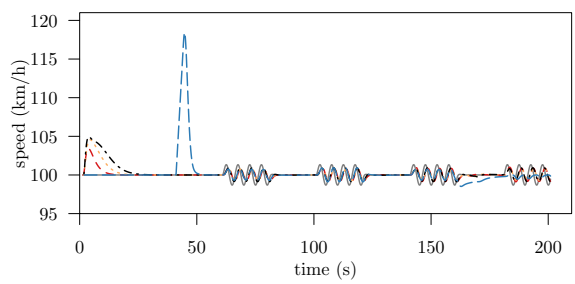

(e)

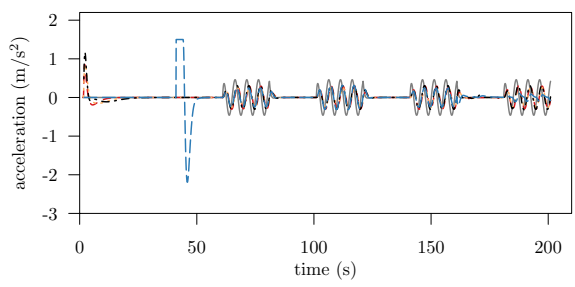

(h)

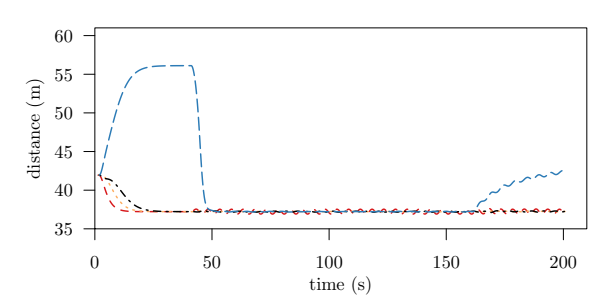

(c)

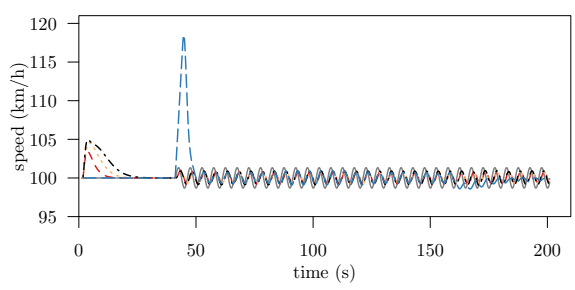

(f)

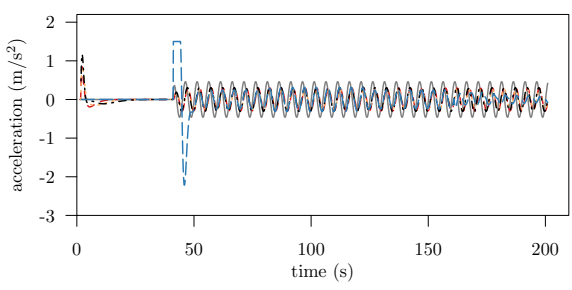

(i)

Figure 4. Join and leave at the tail maneuver: (a), (b), (c) bumper to bumper distance to the vehicle ahead ( $\mathrm{V}_{0}$ not present); (d), (e), (f) vehicles speed; (g), (h), (i) vehicles acceleration. First column no leader disturbance; second and third column sinusoidal disturbances to the leader speed; $T_{h}=40 \mathrm{~s}$.

from the car in front settles to $40 \mathrm{~m}$, concluding the maneuver. Results in Fig. 5 captures the controlled evolution of the entire platoon during transients according to the choice made for the gains values (and hence for the resulting control action). The controller guarantees a very good longitudinal stability during the entire maneuver, showing that it is not necessary to switch to a different longitudinal control technique during the maneuvers, but a single controller can be used for the entire procedure simply switching control topologies.

Finally, Fig. 6 reports the same metrics as Fig. 5, but simulating a definitely harsh scenario, with all the human driven cars beaconing at $10 \mathrm{~Hz}$ and generating interference in the communication process and $T_{h}$ reduced to a mere $10 \mathrm{~s}$. The system shows a remarkably stable behavior under all conditions also with short settling times that enable quick maneuvers.

\section{Leave at Middle}

Finally we consider a vehicle leaving from the middle of the platoon. Figs. 7 and 8 show the results for the maneuver in the standard and realistic scenario, respectively. The maneuver starts with a platoon of five cars; the third car $\left(V_{2}\right)$ wants to leave. The first changes in the topology matrix are not noticeable in the plots, as they simply disable some links without causing changes in the dynamics. When $V_{2}$ leaves the platoon by changing lane, the radar-measured distance of $V_{3}$ from the vehicle in front (now $V_{1}$ ) increases from $40 \mathrm{~m}$ to $80 \mathrm{~m}$. In the final part of the maneuver, the leader communicates to vehicles $V_{3}$ and $V_{4}$ their position change to become the third and fourth vehicle, respectively, switching the matrices accordingly. This causes the last two vehicles to speed up and close the gap left by the leaving vehicle.

\section{CONCLUSION}

Platooning requires that proper maneuvers are enabled within the system to let vehicles join and leave the platoon, besides reacting to external disturbs and emergencies. Protocols to enable this maneuvers need standardization, but the algorithms that realize the semantics of the protocols must be carefully studied to guarantee the safety of passengers. In this paper we have analyzed and explored the use of a distributed consensus algorithm to maintain stability in the platoon while maneuvers are performed and the topology of the platoon changes. The possibility of exploiting the same controller to maintain string stability and to perform maneuvers is a very interesting possibility that to the best of our knowledge has never been proposed before. We have proven the theoretical stability of the platoon under the maneuvers conditions and we have implemented the resulting protocol semantics in PLEXE [24] exploring the performance of the system under realistic conditions (non linear behaviors, network contentions, packet losses, etc.) confirming the theoretical results.

\section{REFERENCES}

[1] B. van Arem, C. van Driel, and R. Visser, "The Impact of Cooperative Adaptive Cruise Control on Traffic-Flow Characteristics," IEEE Trans. on Intelligent Transportation Systems, vol. 7, no. 4, pp. 429-436, Dec. 2006. 


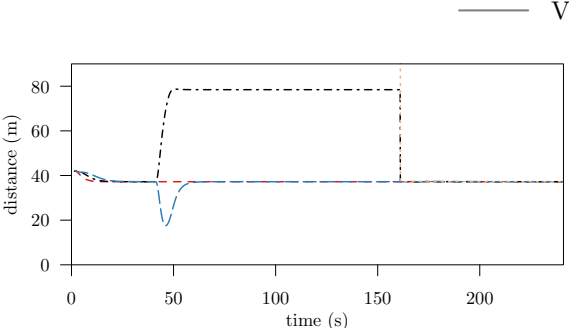

(a)

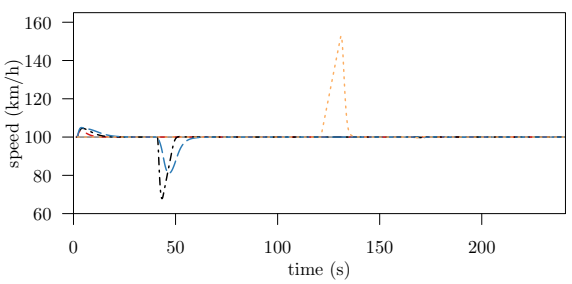

(d)

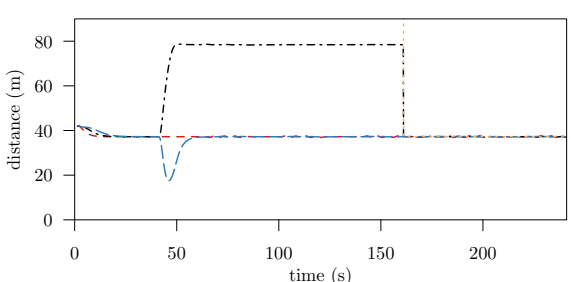

(b)

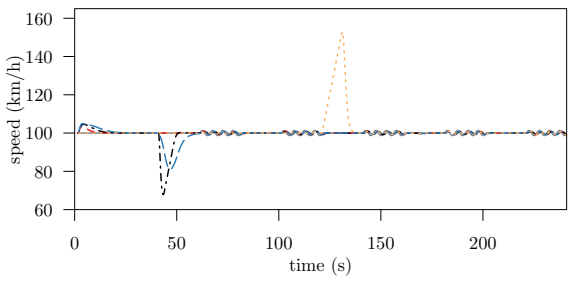

(e)

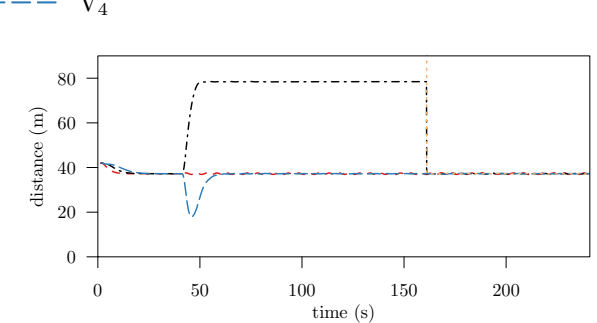

(c)

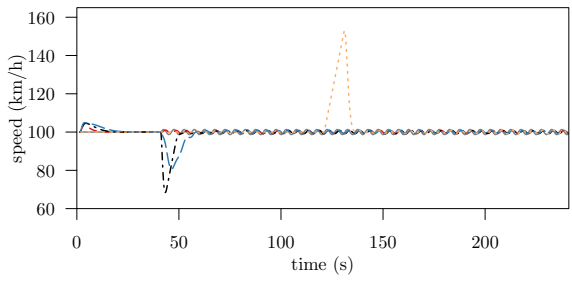

(f)

Figure 5. Join at middle maneuver: (a), (b), (c) bumper to bumper distance to the vehicle ahead ( $\mathrm{V}_{0}$ not present); (d), (e), (f) vehicles speed. First column no leader disturbance; second and third column sinusoidal disturbances to the leader speed; $T_{h}=40 \mathrm{~s}$.

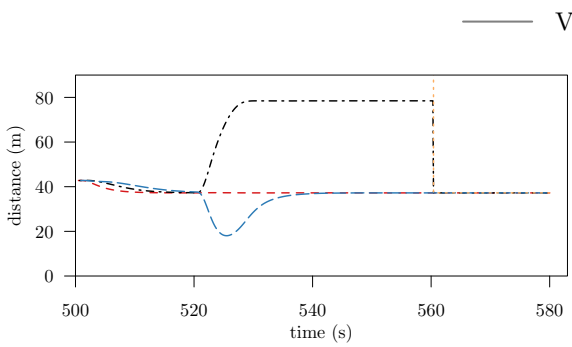

(a)

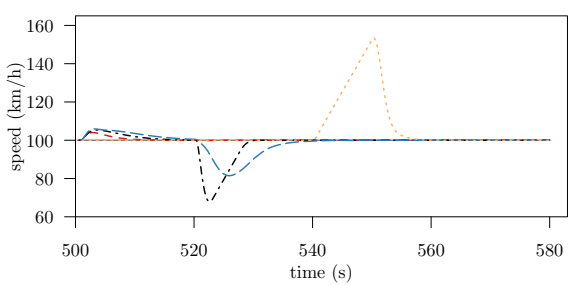

(d)

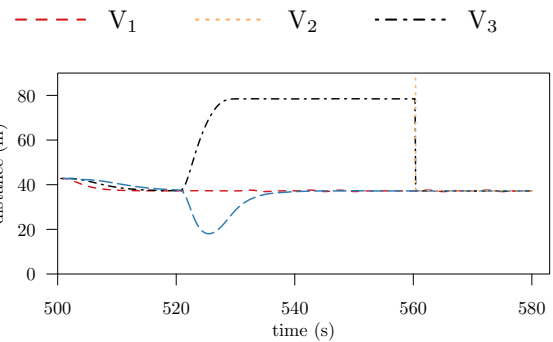

(b)

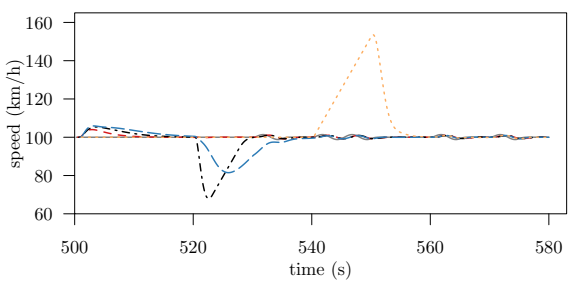

(e)

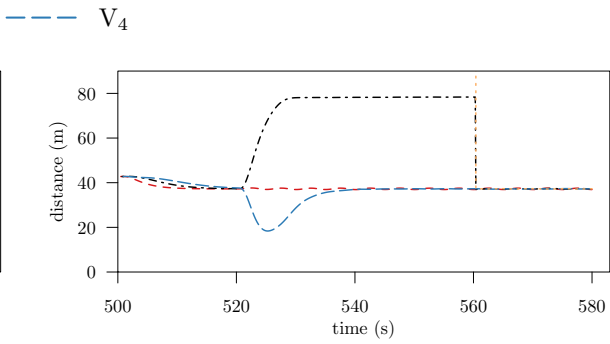

(c)

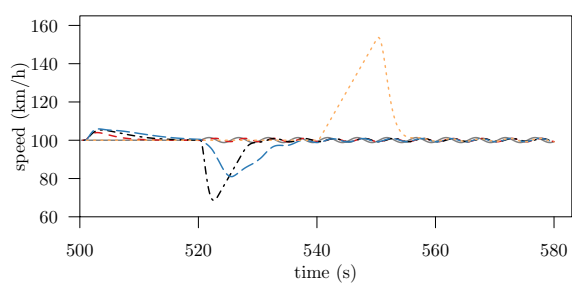

(f)

Figure 6. Join at middle maneuver with realistic interference: (a), (b), (c) bumper to bumper distance to the vehicle ahead (V $\mathrm{V}_{0}$ not present); (d), (e), (f) vehicles speed. First column no leader disturbance; second and third column sinusoidal disturbances to the leader speed; $T_{h}=10 \mathrm{~s}$.

[2] P. S. Jootel, "SAfe Road TRains for the Environment," SARTRE Project, Final Project Report, Oct. 2012.

[3] V. Turri, "Fuel-efficient and Safe Heavy-duty Vehicle Platooning Through Look-ahead Control,' PhD Thesis, KTH Royal Institute of Technology, Oct. 2015.

[4] S. Tsugawa, S. Jeschke, and S. E. Shladover, "A review of truck platooning projects for energy savings," IEEE Transactions on Intelligent Vehicles, vol. 1, no. 1, pp. 68-77, March 2016.

[5] M. Segata, R. Lo Cigno, H.-M. Tsai, and F. Dressler, "On Platooning Control using IEEE 802.11p in Conjunction with Visible Light Communications," in 12th IEEE/IFIP Conf. on Wireless On demand Network Systems and Services (WONS 2016), Cortina d'Ampezzo, Italy, Jan. 2016 pp. 124-127.

[6] M. Segata, "Safe and Efficient Communication Protocols for Platooning Control," PhD Thesis, University of Innsbruck; University of Trento, February Feb. 2016.

[7] S. Joerer, B. Bloessl, M. Segata, C. Sommer, R. Lo Cigno, A. Jamalipour, and F. Dressler, "Enabling Situation Awareness at Intersections for IVC Congestion Control Mechanisms," IEEE Trans. on Mobile Computing vol. 15, no. 7, pp. 1674-1685, Jun. 2016.

[8] B. Paden, M. ?p, S. Z. Yong, D. Yershov, and E. Frazzoli, "A survey of motion planning and control techniques for self-driving urban vehicles,' IEEE Transactions on Intelligent Vehicles, vol. 1, no. 1, pp. 33-55, March 2016.

[9] S. E. Li, Q. Guo, S. Xu, J. Duan, S. Li, C. Li, and K. Su, "Performance enhanced predictive control for adaptive cruise control system considering road elevation information," IEEE Transactions on Intelligent Vehicles, vol. 2, no. 3, pp. 150-160, Sept 2017.

[10] R. Rajamani, H.-S. Tan, B. K. Law, and W.-B. Zhang, "Demonstration of Integrated Longitudinal and Lateral Control for the Operation of Automated Vehicles in Platoons," IEEE Trans. on Control Systems Technology, vol. 8, no. 4, pp. 695-708, July 2000.

[11] J. Ploeg, B. Scheepers, E. van Nunen, N. van de Wouw, and H. Nijmeijer, "Design and Experimental Evaluation of Cooperative Adaptive Cruise Control," in 14th IEEE Int. Conf. on Intelligent Transportation Systems (ITSC 2011), Washington, DC, October 2011, pp. 260-265.

[12] S. Santini, A. Salvi, A. S. Valente, A. Pescapé, M. Segata, and R. Lo Cigno, "A Consensus-Based Approach for Platooning with InterVehicular Communications and Its Validation in Realistic Scenarios,' IEEE Trans. on Vehicular Technology, vol. 66, no. 3, pp. 1985-1999, Mar. 2017.

[13] A. Ali, G. Garcia, and P. Martinet, "The Flatbed Platoon Towing 


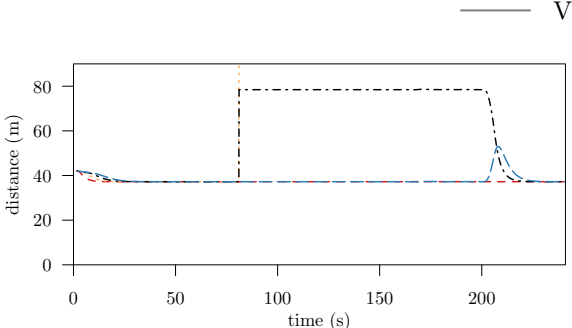

(a)

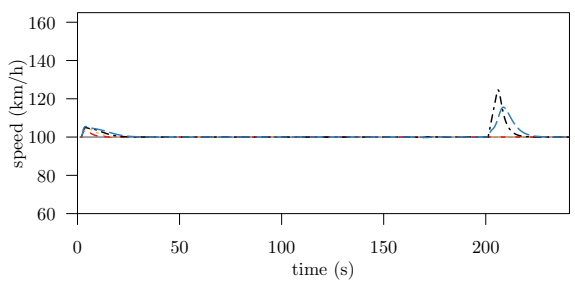

(d)

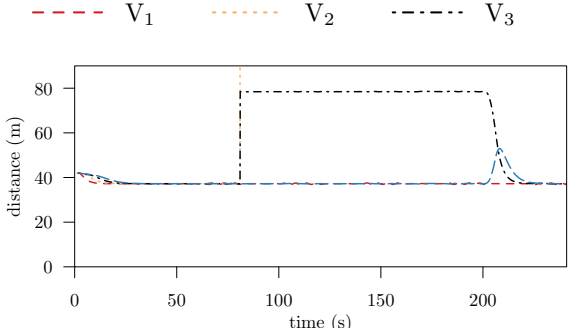

(b)

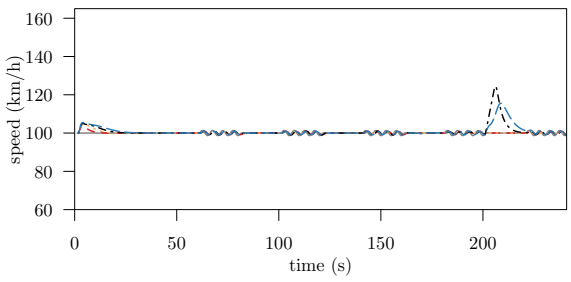

(e)

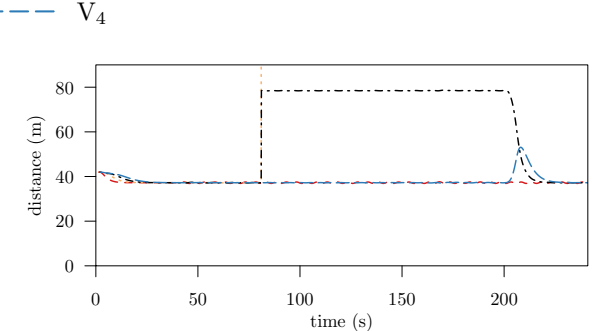

(c)

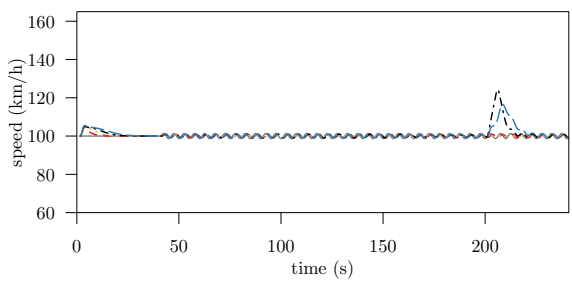

(f)

Figure 7. Leave at middle maneuver: (a), (b), (c) bumper to bumper distance to the vehicle ahead ( $\mathrm{V}_{0}$ not present); (d), (e), (f) vehicles speed. First column no leader disturbance; second and third column sinusoidal disturbances to the leader speed; $T_{h}=40 \mathrm{~s}$.

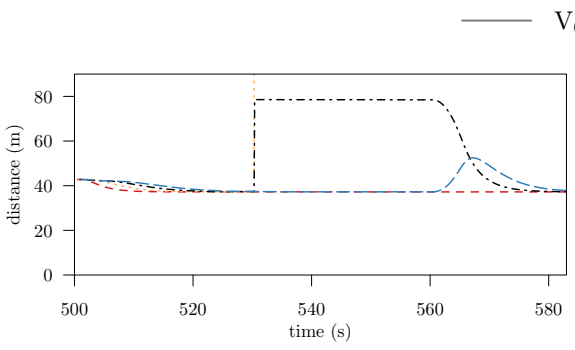

(a)

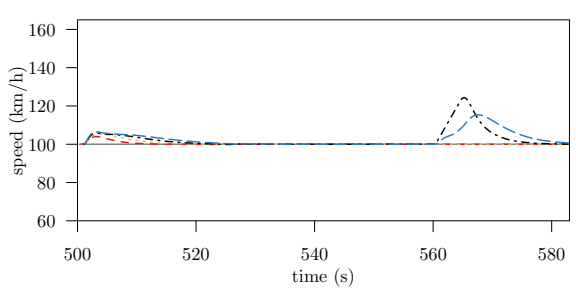

(d)

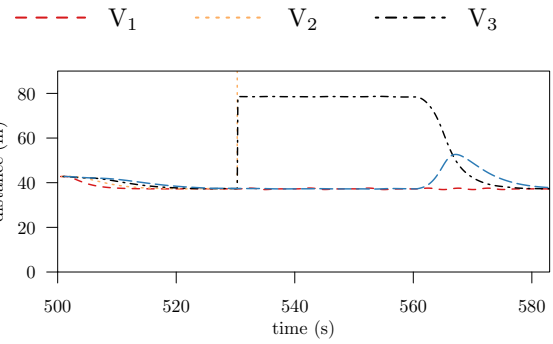

(b)

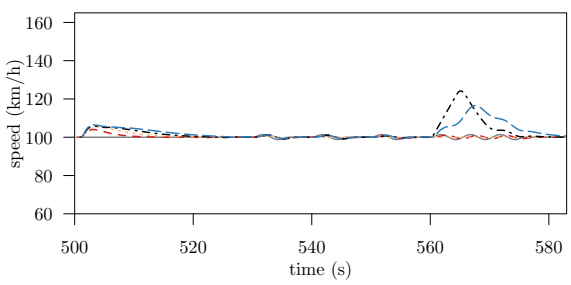

(e)

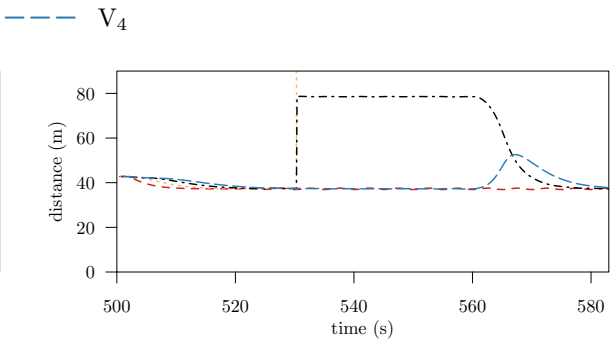

(c)

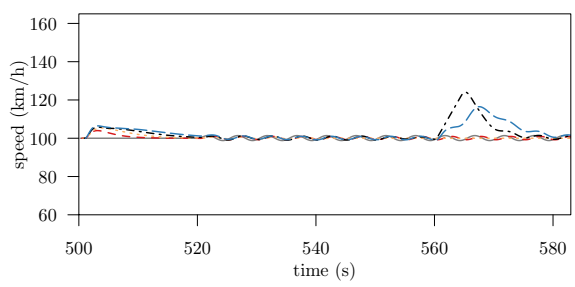

(f)

Figure 8. Leave at middle maneuver with realistic interference: (a), (b), (c) bumper to bumper distance to the vehicle ahead ( $\mathrm{V}_{0}$ not present); (d), (e), (f) vehicles speed. First column no leader disturbance; second and third column sinusoidal disturbances to the leader speed; $T_{h}=10 \mathrm{~s}$.

Model for Safe and Dense Platooning on Highways," IEEE Intelligent Transportation Systems Magazine, vol. 7, no. 1, pp. 58-68, Jan. 2015.

[14] V. Milanés, S. E. Shladover, J. Spring, C. Nowakowski, H. Kawazoe, and M. Nakamura, "Cooperative Adaptive Cruise Control in Real Traffic Situations," IEEE Trans. on Intelligent Transportation Systems, vol. 15 no. 1, pp. 296-305, Feb. 2014.

[15] R. Attia, R. Orjuela, and M. Basset, "Combined longitudinal and lateral control for automated vehicle guidance," Vehicle System Dynamics, vol. 52, no. 2, pp. 261-279, 2014.

[16] T. V., A. Carvalho, H. E. Tseng, K. H. Johansson, and F. Borrelli, "Linear model predictive control for lane keeping and obstacle avoidance on low curvature roads," in 16th IEEE Int. Conf. on Intelligent Transportation Systems (ITSC 2013), Oct 2013, pp. 378-383.

[17] L. Menhour, B. D'Andréa-Novel, M. Fliess, and H. Mounier, "Multivariable decoupled longitudinal and lateral vehicle control: A model-free design," in 52nd IEEE Annual Conference on Decision and Control (CDC), 2013, pp. 2834-2839.

[18] W. Yang, A. Bertozzi, and X. Wang, "Stability of a Second Order Consensus Algorithm with Time Delay," in IEEE CDC, Cancun, Mexico, Dec. 2008, pp. 2926-2931.

[19] M. Segata, B. Bloessl, S. Joerer, C. Sommer, M. Gerla, R. Lo Cigno, and F. Dressler, "Towards Communication Strategies for Platooning: Simulative and Experimental Evaluation," IEEE Trans. on Vehicular Technology, vol. 64, no. 12, pp. 5411-5423, Dec. 2015.

[20] S. Shladover, "PATH at 20 - History and Major Milestones," in IEEE Intelligent Transportation Systems Conference (ITSC 2006), Toronto, Canada, September 2006, pp. 22-29.

[21] S. Santini, A. Salvi, A. S. Valente, A. Pescapè, M. Segata, and R. Lo Cigno, "A Consensus-based Approach for Platooning with Inter-Vehicular Communications," in 34th IEEE Conf. on Computer Communications (INFOCOM 2015), Hong Kong, Apr. 2015, pp. 11581166.

[22] D. Bevly, X. Cao, M. Gordon, G. Ozbilgin, D. Kari, B. Nelson, J. Woodruff, M. Barth, C. Murray, A. Kurt, K. Redmill, and U. Ozguner, "Lane change and merge maneuvers for connected and automated vehicles: A survey," IEEE Transactions on Intelligent Vehicles, vol. 1, no. 1, pp. 105-120, March 2016.

[23] D. Liberzon, Switching in systems and control. Springer Science \& Business Media, 2012.

[24] M. Segata, S. Joerer, B. Bloessl, C. Sommer, F. Dressler, and R. Lo Cigno, "PLEXE: A Platooning Extension for Veins," in 6th IEEE Vehicular Networking Conf. (VNC 2014), Paderborn, Germany, Dec. 2014, pp. 
53-60.

[25] R. Horowitz and P. Varaiya, "Control Design of an Automated Highway System," Proc. of the IEEE, vol. 88, no. 7, pp. 913-925, July 2000.

[26] F. Michaud, P. Lepage, P. Frenette, D. Letourneau, and N. Gaubert, "Coordinated Maneuvering of Automated Vehicles in Platoons," IEEE Trans. on Intelligent Transportation Systems, vol. 7, no. 4, pp. 437-447, Dec. 2006.

[27] S. Hallé, J. Laumonier, and B. Chaib-Draa, "A Decentralized Approach to Collaborative Driving Coordination," in 7th IEEE Intelligent Transportation Systems Conf. (ITSC 2004), Oct. 2004, pp. 453-458.

[28] T. Sakaguchi, A. Uno, S. Kato, and S. Tsugawa, "Cooperative Driving of Automated Vehicles with Inter-Vehicle Communications," in IEEE Intelligent Vehicles Symposium (IV 2000), Dearborn, Michigan, Oct. 2000, pp. $516-521$.

[29] D. Godbole, J. Lygeros, E. Singh, A. Deshpande, and A. Lindsey, "Communication Protocols for a Fault-tolerant Automated Highway System," IEEE Trans. on Control Systems Technology, vol. 8, no. 5, pp. 787-800, Sept. 2000.

[30] R. Rajamani, A. Howell, C. Chen, J. Hedrick, and M. Tomizuka, "A Complete Fault Diagnostic System for Automated Vehicles Operating in a Platoon," IEEE Trans. on Control Systems Technology, vol. 9, no. 4, pp. 553-564, July 2001.

[31] J. Ploeg, E. Semsar-Kazerooni, G. Lijster, N. van de Wouw, and H. Nijmeijer, "Graceful Degradation of CACC Performance Subject to Unreliable Wireless Communication," in 16th IEEE Int. Conf. on Intelligent Transportation Systems (ITSC 2013), The Hague, The Netherlands, Oct. 2013.

[32] M. Segata, B. Bloessl, S. Joerer, F. Dressler, and R. Lo Cigno, "Supporting platooning maneuvers through IVC: An initial protocol analysis for the JOIN maneuver," in 11th IFIP/IEEE Conf. on Wireless On-demand Network Systems and Services (WONS), Apr. 2014, pp. 130-137.

[33] C. Guo, N. Wan, S. Mita, and M. Yang, "Self-defensive Coordinated Maneuvering of an Intelligent Vehicle Platoon in Mixed Traffic," in 15th IEEE Conf. on Intelligent Transportation Systems (ITSC 2012), Anchorage, AK, Sept. 2012, pp. 1726-1733.

[34] S. Lam and J. Katupitiya, "Cooperative Autonomous Platoon Maneuvers on Highways," in IEEE/ASME Int. Conf. on Advanced Intelligent Mechatronics (AIM 2013), Wollogong, Australia, July 2013, pp. 11521157.

[35] M. Segata and R. Lo Cigno, "Automatic Emergency Braking - Realistic Analysis of Car Dynamics and Network Performance," IEEE Trans. on Vehicular Technology, vol. 62, no. 9, pp. 4150-4161, Oct. 2013.

[36] C. Sommer, R. German, and F. Dressler, "Bidirectionally Coupled Network and Road Traffic Simulation for Improved IVC Analysis," IEEE Trans. on Mobile Computing, vol. 10, no. 1, pp. 3-15, Jan. 2011.

[37] M. Segata, F. Dressler, R. Lo Cigno, and M. Gerla, "A simulation tool for automated platooning in mixed highway scenarios," in 18th ACM Int. Confon Mobile Computing and Networking (MobiCom'12), Istanbul, Turkey, Aug. 2012, pp. 389-392.

[38] R. A. Horn and C. R. Johnson, Matrix Analisis. Cambridge: University Press, 1987.

[39] J. Hu and Y. Hong, "Leader-following coordination of multi-agent systems with coupling time delays," Physica A: Statistical Mechanics and its Applications, vol. 374, no. 2, pp. 853 - 863, 2007.

[40] U. Munz, A. Papachristodoulou, and F. Allgower, "Consensus in multiagent systems with coupling delays and switching topology," IEEE Trans. on Automatic Control, vol. 56, no. 12, pp. 2976-2982, Dec. 2011.

[41] D. Hershkowitz, "Recent directions in matrix stability," Linear Algebra and its Applications, vol. 171, pp. 161 - 186, 1992.

[42] R. Horn and C. Johnson, "Topics in matrix analysis," Cambridge University Presss, Cambridge, 1991.

[43] T.-Z. Huang and Y. Zhu, "Linear algebra and its applications," Elsivier, vol. 432, no. 2, pp. 670-677, 2010

[44] P. Shivakumar, J. J. Williams, Q. Ye, and C. A. Marinov, "On twosided bounds related to weakly diagonally dominant m-matrices with application to digital circuit dynamics," SIAM Journal on Matrix Analysis and Applications, vol. 17, no. 2, pp. 298-312, 1996.

[45] S. Darbha and K. Rajagopal, "Intelligent Cruise Control Systems and Traffic Flow Stability," Elsevier Transp. Research Part C: Emerging Technologies, vol. 7, no. 6, pp. 329-352, Dec 1999.

[46] P. Fernandes and U. Nunes, "Platooning With IVC-Enabled Autonomous Vehicles: Strategies to Mitigate Communication Delays, Improve Safety and Traffic Flow," IEEE Transactions on Intelligent Transportation Systems, vol. 13, no. 1, pp. 91-106, March 2012.

[47] J. K. Hale and S. M. V. Lunel, Introduction to Functional Differential Equations. Springer-Verlag, 1993.
[48] Z. Wei and C. Daizhan, "Leader-following consensus of second-order agents with multiple time-varying delays," Automatica, vol. 46, no. 12, pp. $1994-1999,2010$.

[49] A. F. Filippov, Differential Equations with Discontinuous Righthand Sides. Kluwer Academic Publishers, 1988.

[50] K. Liu, G. Xie, and L. Wang, "Consensus for multi-agent systems under double integrator dynamics with time-varying communication delays," International Journal of Robust and Nonlinear Control, vol. 22, no. 17, pp. 1881-1898, 2012

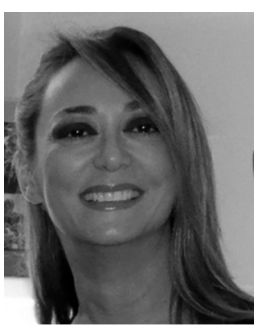

Stefania Santini is an Associate Professor of automatic control with the Department of Electrical Engineering and Information Technology, University of Naples Federico II. Stefania Santini received the M.Sc. degree in electronic engineering and the Ph.D. degree in automatic control from the University of Naples Federico II, Naples, Italy, in 1996 and 1999 respectively. Dr. Santini's Ph.D. research work was supported by the Engine Institute of the National Research Council. She is involved in many projects with industry, including small- and medium-sized enterprises (SMEs) operating in the automotive field. Her research interests include the area of the analysis and control of non-linear systems with applications to automotive engineering, transportation technologies, and, more recently, computational biology.

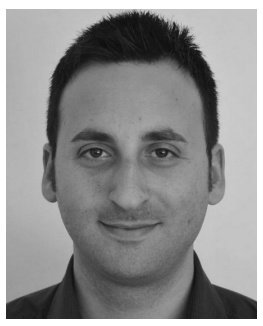

Alessandro Salvi received the M.Sc. degree in control systems engineering and the Ph.D. degree in computer science and control systems engineering, with the thesis entitled "Cooperative Control for Vehicle Platooning: A Complex Network Approach," from the University of Naples Federico II, Naples, Italy, in 2010 and 2014, respectively. He was a Visiting Ph.D. student with Universitat Politècnica de Catalunya, Barcelona, Spain, in 2011 and with the Chalmers University of Technology, Göteborg, Sweden, in 2013. He is currently with the Department of Electrical Engineering and Information Technology, University of Naples Federico II. His research interests include the distributed control of complex networks with applications to automotive engineering and power systems.

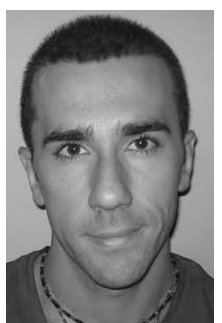

Antonio Saverio Valente received his M.Sc. degree in Control Systems Engineering in 2010. In 2015 he got the Ph.D. degree in Computer Science and Control Systems engineering from the University of Naples Federico II, with the thesis entitled "Cooperative Driving in Inter-Vehicular Communication Network". He is currently with the Department of Electrical Engineering and Information Technology (DIETI), University of Naples Federico II. His research interests focus on the distributed control of complex networks with applications to automotive engineering and on the monitoring of fleets of vehicles via floating car data. 


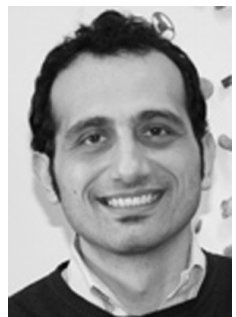

Antonio Pescapé [SM '09] is a Full Professor at the Department of Electrical Engineering and Information Technology of the University of Napoli Federico II (Italy) where he teaches courses in Computer Networks, Computer Architectures, Programming, and Multimedia and he has also supervised and graduated more than 160 among $\mathrm{BS}, \mathrm{MS}$, and $\mathrm{PhD}$ students. His research interests are in the networking field with focus on Internet Monitoring, Measurements and Management and on Network Security. Antonio Pescapé has co-authored over 170 journal (IEEE ACM Transaction on Networking, Communications of the ACM, IEEE Communications Magazine, JSAC, IEEE Wireless Communications Magazine, IEEE Networks, etc.) and conference (SIGCOMM, NSDI, Infocom, Conext, IMC, PAM, Globecom, ICC, etc.) publications and he is co-author of a patent $\mathrm{He}$ has served and serves as workshops and conferences Chair (including IEEE ICC (NGN symposium)) and on more than 170 technical program committees of IEEE and ACM conferences. For his research activities he has received several awards, comprising a Google Faculty Award, several best paper awards and two IRTF (Internet Research Task Force) ANRP (Applied Networking Research Prize). Antonio Pescapé has served and serves as independent reviewer/evaluator of research and implementation projects and project proposals co-funded by the EU Commission, Sweden government, several Italian local governments, Italian Ministry for University and Research (MIUR) and Italian Ministry of Economic Development (MISE). Antonio Pescapé is a Senior Member of the IEEE.

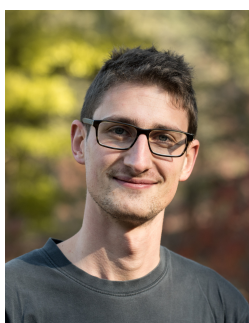

Michele Segata received his B.Sc. and M.Sc. in Computer Science from the University of Trento in 2009 and 2011, respectively. In 2016 he got a double $\mathrm{PhD}$ in Computer Science from the Universities of Innsbruck and Trento. His research mainly focuses on the development of communication protocols and highly realistic simulation models for platooning. He also worked on vehicular networking-based safety application and a software defined radio implementation of the IEEE $802.11 \mathrm{p}$ physical layer. He volunteered in the organization of international conferences such as VNC and WONS. Since April 2016 he is a Postdoctoral Research Fellow with the Advanced Networking Systems group in Trento, leaded by Prof. Renato Lo Cigno.

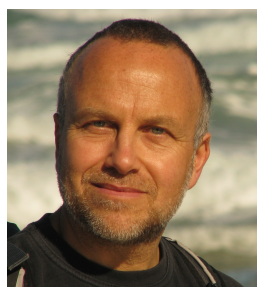

Renato Lo Cigno (SM'11) is an Associate Professor at the Department of Computer Science and Telecommunications (DISI) of the University of Trento, Italy, where he leads the Advanced Networking Systems research group. He received a degree in Electronic Engineering with a specialization in Telecommunications from Politecnico di Torino in 1988, the same institution where he worked until 2002. From June 1998 to February 1999, he was with the Computer Science Department, University of California, Los Angeles, as Visiting Scholar. His current research interests are in the modeling, design, and performance evaluation of wired and wireless networks, vehicular networks, networked systems including cooperative on-line systems. Renato Lo Cigno has been Area Editor for Computer Networks for five years, and he is now editor for IEEE Transactions on Communications. He has been involved in the organization of several IEEE and ACM Conferences, including Infocom, Peer to Peer Networks (P2P) and Vehicular Networking Conference (VNC). He is the Chair of the Steering Committee of WONS (Wireless On-demand Networks and Services), and has been awarded six Best Paper Award in IEEE or ACM sponsored conferences. $\mathrm{He}$ is senior member of IEEE and ACM and has co-authored about 200 papers all in international, peer reviewed venues. 